\title{
Integrale Quantifizierung von Schadstoffmassenflüssen im Grundwasser - Konditionierung der numerischen Inversion der Konzentrationsganglinien von Immissionspumpversuchen
}

\author{
Maria Herold · Thomas Ptak • Thomas Wendel · Peter Grathwohl
}

Eingang des Beitrages: 23.7.2007/Eingang des überarbeiteten Beitrages: 2.11.2007/Online veröffentlicht: 8. 1.2008

(C) Die Autoren 2008

Kurzfassung Auf dem Gelände eines ehemaligen Gaswerks (Testfeld Süd) wurden Immissionspumpversuche (IPVs) zur Quantifizierung der Fracht und der mittleren Konzentration gaswerkstypischer Schadstoffe entlang von drei Kontrollquerschnitten durchgeführt. Die daraus resultierenden Konzentrationsganglinien wurden mithilfe des Inversionsprogramms CSTREAM und eines Strömungsund Transportmodells des hydraulisch extrem heterogenen Grundwasserleiters numerisch ausgewertet. Die den gesamten Abstrombereich der Verdachtsfläche erfassenden Kontrollquerschnitte ermöglichen Aussagen über die Position und Ausbreitung der Schadstofffahne auf dem Gelände des Testfeldes Süd. Bisherige Auswertungen von IPVs konnten die Verteilung der Schadstofffahne um den IPV-Brunnen für genau drei Fälle berechnen: entweder befindet sich die Fahne links vom Brunnen, rechts davon oder ist symmetrisch um ihn verteilt. Um eine realistischere Vorstellung von der Fahnenposition zu ermöglichen, wurden in dieser Studie zusätzlich Direct-Push-Messstellen entlang einer Kontrollebene installiert. Die in diesen Messstellen gemessenen Konzentrationen wurden zur Konditionierung der numerischen Inversionslösung herangezogen. Die Ergebnisse ermöglichen eine genauere Eingrenzung des Fahnenzentrums sowie des Fahnenrandes, was insbesondere die Erarbeitung angepasster und optimierter Sanierungsstrategien unterstützt.

M.Sc. M. Herold, Prof. Dr.-Ing. T. Ptak

Angewandte Geologie, Geowissenschaftliches Zentrum, Universität Göttingen, Goldschmidtstr. 3, 37077 Göttingen

E-Mail: mherold@gwdg.de, thomas.ptak@geo.uni-goettingen.de

Dr. T. Wendel, Prof. Dr. P. Grathwohl

Zentrum für Angewandte Geowissenschaften, Universität Tübingen, Sigwartstr. 10, 72076 Tübingen

E-Mail: thomas.wendel@uni-tuebingen.de,peter.grathwohl@unituebingen.de
Integral quantification of contaminant mass flux in groundwater - conditioning of the numerical inversion of concentration-time series of integral pumping tests

\begin{abstract}
A series of integral pumping tests (IPTs) were conducted at a former gasworks site to quantify the contaminant mass flux and average concentration in groundwater along three control planes. The resulting concentration-time series were analysed numerically with the help of the inversion code CSTREAM and a flow and transport model representing the highly heterogeneous aquifer. Since the control planes cover the entire downstream width of the potentially contaminated area, they allow conclusions to be drawn about the current location and spread of the contaminant plume. Previous evaluations of integral pumping tests could calculate three scenarios concerning the spread of the plume around the IPT well: (i) the plume is located to the right of the well, (ii) to the left, or (iii) is distributed symmetrically around it. To create a more realistic picture of the plume position, a series of direct-push monitoring wells were installed along one control plane. The concentrations found in these wells were included in the numerical analysis to condition the inversion results, and resulted in a more pronounced plume centre and fringe, which supports the development of optimised remediation strategies.
\end{abstract}

Keywords integral pumping tests $\cdot$ conditioning $\cdot$ plume delineation $\cdot$ remediation optimisation

\section{Einleitung}

Das Herangehen an großräumige Schadensfälle unter Berücksichtigung von Natural Attenuation (NA) hat in den letzten Jahren mehr und mehr an Bedeutung gewonnen, da im Vergleich zu Sanierungsmaßnahmen wie z. B. Pump-and- 
Treat die Kosten deutlich besser eingrenzbar sind und in der Summe auch niedriger ausfallen können. Die Betrachtung von NA bedingt aber erstens die Dokumentation der Abnahme des Schadstoffmassenflusses im Untersuchungsgebiet, zweitens die Darstellung von Tendenzen bei hydrogeochemisch relevanten Parametern, wie die $\mathrm{Zu}$ - und Abnahme von Abbauprodukten und Elektronenakzeptoren, und drittens den Nachweis mikrobieller Aktivität vor Ort (Nyer \& Duffin 1997, vgl. auch LABO-Arbeitspapier „Berücksichtigung natürlicher Schadstoffminderungsprozesse bei der Altlastenbearbeitung“", 2005). Hierbei ist die Abnahme des Schadstoffmassenflusses der am schwierigsten zu erbringende Beweis. Erst mit dieser Informationsgrundlage kann es zu einer Einschätzung und Bewertung des Schadensfalls sowie der genauen Konzeption, Positionierung und Dimensionierung von Sanierungsmaßnahmen und der Berücksichtigung von NA kommen.

Der jeweilige Nachweis von NA ist deshalb entscheidend, da NA vollständig von den Bedingungen vor Ort kontrolliert wird. Sowohl die Zusammensetzung und Heterogenität der Quelle als auch die inhärente Heterogenität des betroffenen Grundwasserleiters sind ausschlaggebend für die Freisetzung und Verbreitung des Schadstoffs. Beide Faktoren prägen die Ausbreitung und die Konzentration des Schadstoffes im Grundwasserleiter maßgeblich, sind aber gleichzeitig in der Regel am wenigsten untersucht und quantifiziert. Letzteres ist darauf zurückzuführen, dass die bisher angewandten Erkundungsmethoden, z. B. räumliches und zeitliches Monitoring von Schadstoffkonzentrationen und Elektronenakzeptoren (Chiang et al. 1989, Gieg et al. 1999), Tracerversuche mit isotopen-markierten Schadstoffen (Therrin et al. 1995) oder abbauresistenten Tracern (Wiedemeier et al. 1996), Messungen von verbindungsspezifischen stabilen Isotopenverhältnissen (Hunkeler et al. 1999) oder die Berechnung des Massenflusses an Kontrollebenen (King et al. 1999, Borden et al. 1997), nur ein partielles Bild eines Untergrundes mit seiner Schadstoffverteilung wiedergeben konnten, da alle diese Methoden auf dem Ansatz der Interpolation von Punktkonzentrationsmessungen basieren. Die Heterogenität des Bodens führt zu sehr unregelmäßig ausgebildeten Schadstofffahnen, deren Abgrenzung bei der Erkundung stark von der Anzahl und Positionierung der Monitoring-Brunnen abhängt. Weiterhin führt die heterogene Verteilung des Schadstoffs im Herd zu einer ebenso ungleichmäßig verteilten Schadstoffkonzentration im Abstrom. Nur ein ausreichend dichtes Messstellennetz könnte sicherstellen, dass die Fahne in ihrem ganzen Ausmaß erfasst wird. Da eine solche Erkundung schnell an ihre finanziellen Grenzen stößt und trotzdem keine hundertprozentige Erkundungssicherheit bietet, wurde eine räumlich integrierende Erkundungsmethode entwickelt, die so genannten Immissionspumpversuche (,IPV“: Teutsch et al. 2000, Ptak et al. 2000), die, angeordnet entlang von einer oder mehrerer Kontrollebenen, im Abstrom des Schadensherdes durchgeführt werden. Durch das große Erfassungsvolumen an den einzelnen IPV-Brunnen reduziert sich die aus der räumlichen Konzentrationsvariabilität resultierende Erkundungsunsicherheit. Eine richtig positionierte und dimensionierte Kontrollebene kann die gesamte Fahne erfassen. Durch Anwendung eines Inversionsalgorithmus auf gemessene IPV-Schadstoffkonzentrationsganglinien erhält man dann die gesamte Schadstofffracht und die mittlere Schadstoffkonzentration an der Kontrollebene. Die Inversion kann analytisch, unter Berücksichtigung der natürlichen Grundwasserströmung (Bayer-Raich et al. 2004), und numerisch, unter Einbindung eines Grundwasserströmungsund Transportmodells (Bayer-Raich et al. 2003), erfolgen. Beispiele für solche Auswertungen findet man unter anderem bei Bockelmann et al. (2001, 2003), Bauer et al. (2004) und Rügner et al. (2004), wobei Ptak et al. (2004) eine höhere räumliche Auflösung durch vertikale Differenzierung der IPV-Konzentrationsganglinien erreicht haben. Durch die Nutzung von zwei oder mehreren, im Abstrom hintereinander liegenden Kontrollebenen, kann dabei auch die NA-Rate bestimmt werden, wie im Falle von Benzol, Toluol, Ethylbenzol und Xylol (BTEX) und Polyaromatischen Kohlenwasserstoffen (PAKs) bereits veranschaulicht wurde (Bockelmann et al. 2001, Bayer-Raich et al. 2006).

Die Auswertung eines IPVs hatte jedoch bisher den Nachteil, dass die Berechnung der Schadstofffracht nur für drei bestimmte Fälle möglich war: entweder wird eine symmetrische Verteilung der Fahne um den Brunnen herum angenommen oder sie fließt entweder rechts oder links am Brunnen vorbei (z.B. Jarsjö et al. 2005). Dieses Problem kann mithilfe einer Konditionierung der Inversionslösung mit Punktkonzentrationsmessungen umgangen werden. Dabei werden durch z.B. Direct-Push-Technik gewonnene Informationen bezüglich der Schadstoffkonzentration entlang der Kontrollebene in der Umgebung des IPV-Brunnens zur Auswertung herangezogen. Dies macht eine deutlich genauere und eindeutigere Bestimmung der Fahnenposition möglich.

In der vorliegenden Studie wird diese Herangehensweise erstmals zur exakten Lagebestimmung einer NSO-Heteroaromaten-(HET)-Schadstofffahne an einem ehemaligen Gaswerksstandort (Testfeld Süd, Herfort et al. 1998) getestet. An diesem Standort wurden bereits mithilfe von IPVs ohne Konditionierung die Schadstoffmassenflüsse quantifiziert und als Eingangsgrößen zur Dimensionierung einer innovativen Sanierungsmaßnahme $\left(\mathrm{H}_{2} \mathrm{O}_{2}\right.$-Einmischung mittels Grundwasserzirkulationsbrunnen) verwendet. Die genaue Kenntnis der Fahnenposition ist eine grundlegende Voraussetzung für weitere Studien bzgl. der Schadensherdposition und für die reaktive Schadstofftransportmodellierung im Zusammenhang mit der Sanierungsmaßnahme, welche im Rahmen eines vom BMBF finanzierten KORA (Kontrollierter natürlicher Rückhalt und Abbau von Schad- 
stoffen bei der Sanierung kontaminierter Grundwässer und Böden)-Projektes durchgeführt wird.

\section{Standortbeschreibung}

Hydrogeologie

Das Testfeld Süd befindet sich auf dem Gelände eines ehemaligen Gaswerksstandortes im Neckartal in Süddeutschland und hat eine Nord-Süd-Ausdehnung von ungefähr $1.000 \mathrm{~m}$ und eine Breite zwischen 160 und $400 \mathrm{~m}$. Es wird im Osten durch den Neckar und im Westen durch den Talrand begrenzt.

Der hier untersuchte quartäre Grundwasserleiter mit einer Mächtigkeit von durchschnittlich 3,3 m besteht aus Mittelkies mit örtlich begrenzten feinkörnigen Lagen und wird entweder von Auesedimenten oder anthropogenen Auffüllungen überdeckt. Im Liegenden folgt eine $30 \mathrm{~m}$ mächtige Gipskeuperschicht und daraufhin der Obere Muschelkalk. Erstere stellen die Quelle von sulfatreichem Wasser dar, welches im untersuchten Grundwasserleiter nachgewiesen wurde. Die ausgeprägte Heterogenität des Grundwasserleiters ist durch die Sedimentationsbedingungen vor Ort, die Lage am Talrand (Ausstreichen und Auskeilen der Schichten), lokale Einsenkungen im Festgesteinsuntergrund (Störungen und Auslaugung) sowie anthropogene Einflüsse wie Baumaßnahmen (Aushub, Fundamente) bedingt.

Der gemessene Abstand zwischen Geländeoberkante und Grundwasser beträgt im Mittel 4,1 m, wobei der Grundwasserleiter, abhängig von der Art der Überdeckung (Auesediment oder anthropogene Auffüllung), gespannt bzw. ungespannt ist.

Die gemessenen Grundwasserpotenziale der letzten 10 Jahre schwanken nur geringfügig, und ein saisonaler Trend ist nicht erkennbar. Beides ist auf die regulierende Funktion des künstlich aufgestauten Vorfluters zurückzuführen. Tracerversuche ergaben einen Gradienten von 0,002, wobei die Hauptfließrichtung als N-NW bestimmt wurde (Herfort 2000), und eine Abstandsgeschwindigkeit von $2 \mathrm{md}^{-1}$ (Bösel et al. 2000). Die speichernutzbare Porosität wurde mit drei verschiedenen Verfahren (Abschätzung aus $\mathrm{k}_{\mathrm{f}}$-Werten nach Marotz (1968), Markierungsversuch 1989 und Auslaufversuche an Bohrkernen) bestimmt und ergab Werte von $12-20 \%, 6-7 \%$ bzw. 3-19\% bei einem Mittelwert von 10\%.

Eine große Anzahl von Pumpversuchen im zentralen und südlichen Teil des Geländes ergab einen mittleren $\mathrm{k}_{\mathrm{f}}$-Wert von $3,3 \cdot 10^{-3} \mathrm{~ms}^{-1}$ (Herfort 2000), und aus der hydraulischen Auswertung der Immissionspumpversuche 2004 und 2006 resultierte ein mittlerer $\mathrm{k}_{\mathrm{f}}$-Wert von $3,7 \cdot 10^{-3} \mathrm{~ms}^{-1}$ für den nördlichen Teil.

Das Gaswerk war zwischen 1875 und 1970 in Betrieb und Leckagen in Bereichen der Kohleverarbeitung, insbesondere aber die Zerstörungen im 2. Weltkrieg, haben zu ei- ner großen Anzahl von Schadensherden geführt (Zamfirescu \& Grathwohl 2001). Aus diesem Grund wird das Alter des Hauptschadensherdes auf ungefähr 60 Jahre geschätzt. Das Schadstoffspektrum umfasst gaswerkstypische Substanzen wie monoaromatische Kohlenwasserstoffe, polyzyklische aromatische Kohlenwasserstoffe (PAK), aliphatische Kohlenwasserstoffe, Phenole und heterozyklische aromatische Kohlenwasserstoffe (HET) (Zamfirescu 2000). In zahlreichen Bohrungen im südlichen Teil des Testfeldes wurde an Top und Basis des Grundwasserleiters Öl in Phase gefunden. Weitere Bohrungen im zentralen und nördlichen Teil weisen auf die Ausbildung einer komplexen Schadstofffahne hin. Die vorherrschenden Bedingungen im Grundwasser sind sulfatreduzierend.

\section{Methodik}

Grundlagen Immissionspumpversuche

Immissionspumpversuche (IPVs, Teutsch et al. 2000, Ptak et al. 2000) werden seit einigen Jahren eingesetzt, um die Schadstofffracht unterstromig von Schadstoffquellen zu bestimmen. Hierzu werden Pumpbrunnen entlang einer Kontrollebene angeordnet und entweder gleichzeitig oder sequenziell bepumpt. Die Positionierung der Brunnen sowie deren Pumpraten und Pumpdauer müssen so gewählt werden, dass optimalerweise der gesamte Abstrombereich der Verdachtsfläche erfasst wird. Während der Pumpmaßnahmen werden Konzentrationsganglinien der Schadstoffe und anderer relevanter Grundwasserqualitätsparameter gemessen, die Information über die Position und die Ausmaße der Fahne sowie über die Konzentrationen der Schadstoffe und die Verteilung der Grundwasserparameter geben. Die Konzentrationsganglinien werden dann unter Verwendung eines instationären Inversionsalgorithmus analytisch oder numerisch mithilfe eines Strömungs- und Transportmodells ausgewertet, um Konzentrationsverteilungen entlang der Kontrollebene und die entsprechenden Schadstofffrachten zu erhalten. Dieses Prinzip wird in Abbildung 1 verdeutlicht.

\section{Numerische Auswertung}

Der Inversionsalgorithmus der zur numerischen Inversion der Konzentrationsganglinien verwendet wird, ist in dem Programm CSTREAM (Bayer-Raich et al. 2003, BayerRaich 2004) implementiert, welches eine Erweiterung der Inversionslösung von Schwarz (2002) darstellt. CSTREAM benötigt ein instationäres und advektives Strömungs- und Transportmodell des Untersuchungsgebietes, welches dazu dient, die Stromlinien zu bestimmen. Dieses Modell simuliert alle IPVs unter Berücksichtigung der räumlichen Variabilität der hydraulischen Leitfähigkeit, der Porosität und 
Abb. 1 Prinzip der IPVs (Ptak et al. 2004).

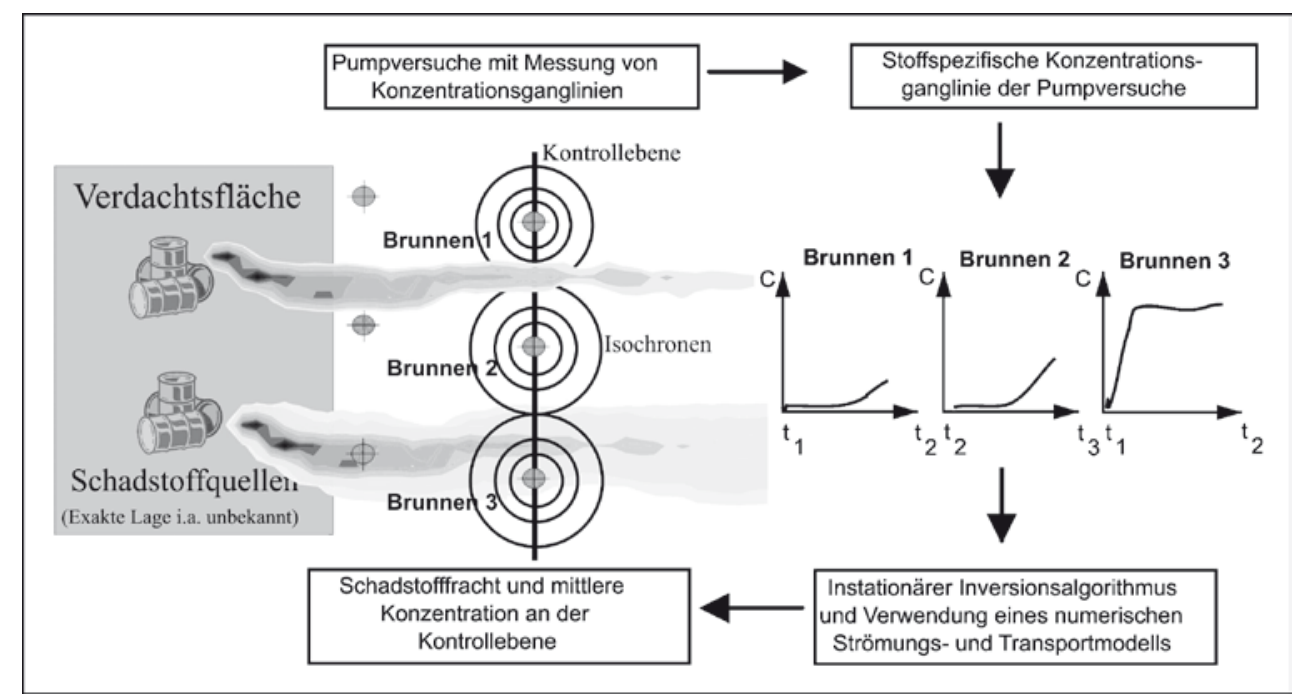

Abb. 2 Definition von Isochronen, Stromlinien und Stromröhren-Konzept in CSTREAM; Fall 1 (Abb. 2a): Symmetrische Inversionslösung; Fall 2 (Abb. 2b): Lösung mit Konditionierung; $11(\mathrm{tn})=$ Isochrone zum Zeitpunkt $\mathrm{n} ; \mathrm{Cn}=$ Konzentration in Isochrone $\mathrm{n} ; \mathrm{Anl} / \mathrm{r}=$ Fläche der Stromröhre, definiert durch Isochronen $\mathrm{n}$ und $\mathrm{n}-1$, links oder rechts vom IPV-Brunnen; für Fall 2 entspricht die Konzentration C4 für A41 der Konzentration CMessstelle (CCZStromröhre), die Konzentration $\mathrm{C} 4$ für A4r resultiert aus der Massenerhaltung.

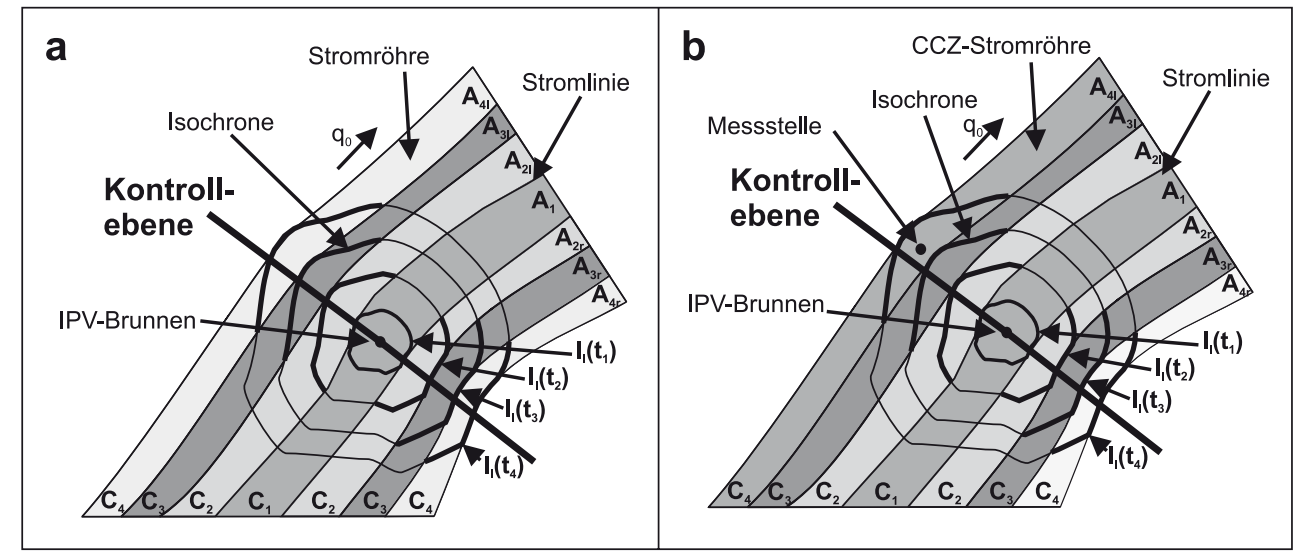

der Mächtigkeit des Grundwasserleiters. Dadurch wird es der numerischen Inversionslösung, im Gegensatz zur analytischen Lösung, ermöglicht, die Einzugsbereiche der IPVs unter Berücksichtigung der Heterogenität des Untergrunds und der Einflüsse des hydraulischen Gradienten abzubilden. Die Schadstofffracht über die Kontrollebene, $M_{C P}\left[\mathrm{M} \mathrm{T}^{-1}\right]$, wird wie folgt berechnet:

$$
M_{C P}(t)=\int_{\ell_{C P}(t)} C_{0}(x, 0) q_{0 y}(x, 0) b(x, 0) d x
$$

wobei $C_{0}(x, 0)$ die Konzentrationsverteilung entlang der Kontrollebene in x-Richtung, $q_{0 y}(x, 0)$ die Darcy-Geschwindigkeit vor der Pumpmaßnahme senkrecht zur Kontrollebene und $b(x, 0)$ die Mächtigkeit des Grundwasserleiters darstellen. Zur Bestimmung der Konzentrationsverteilung werden Isochronen gemäß dem Beprobungsverlauf und dem natürlichen Verlauf der Stromlinien im Modell definiert (Abb. 2). Die Abhängigkeit der Konzentrationsganglinien $\mathrm{C}_{w}(t)$ von der Konzentrationsverteilung vor Beginn der
Pumpmaßnahme $C_{0}(x, y)$ kann durch die folgende Massenbilanz (Bayer-Raich et al. 2004) dargestellt werden:

[2] $\quad Q_{w} C_{w}(t)=-\oint_{\ell_{l}(t)} C_{0}(x, y) \vec{q}_{w}(x, y) \vec{n} b(x, y) d \ell$

wobei $\vec{q}_{w}(x, y)$ [L T $\left.{ }^{-1}\right]$ als Darcy-Geschwindigkeit während des Pumpversuchs, $\vec{n}$ als nach außen zeigender Einheitsvektor rechtwinklig zur Isochrone, $b(x, y)$ [L] als wassergefüllte Aquifermächtigkeit,

$Q_{w}=-\int_{\ell_{l}(t)} \vec{q}_{w}(x, y) \vec{n} b(x, y) d \ell\left[\mathrm{L}^{3} \mathrm{~T}^{-1}\right]$

als Pumprate am Brunnen und $\ell_{l}(t)$ als Isochrone zum Zeitpunkt $t$ definiert ist. Gleichung 2 wird für jeden Wert der Konzentrationsganglinie gelöst, wobei die Konzentrationsverteilung der vorausgegangenen Isochronen mit einbezogen wird.

Die Isochrone $\ell_{l}\left(t_{1}\right)$ in Abbildung 2 zeigt die Herkunftsposition der Grundwasserprobe vor Beginn des Pumpversuchs. Die Inversionslösung für $t_{1}$ ergibt damit eine Kon- 
zentration $C_{1}$ repräsentativ für die Fläche $A_{1}$, die der zu diesem Zeitpunkt gemessenen Konzentration $\left(C_{1}=C_{w}\left(t_{1}\right)\right)$ entspricht. Die Situation stellt sich leicht verändert für die weiteren Isochronen dar. Da angenommen wird, dass die Schadstoffkonzentration innerhalb des Einzugsgebietes der Pumpmaßnahme in Fließrichtung konstant bleibt, wird nur der außerhalb von $A_{1}$ liegende Teil für $\ell_{l}\left(t_{2}\right)$ neue Informationen bereithalten. Gleichung 2 wird für $t_{2}$ gelöst, wobei die zuvor berechnete Konzentration $C_{1}$, welche für die Fläche $A_{1}$ repräsentativ ist, in der Berechnung über eine Massenbilanzierung berücksichtigt wird. Die sich daraus ergebende Konzentration $\left(C_{2}\right)$ gilt sowohl für die sich rechts von $A_{1}$ befindliche Fläche $\left(A_{2 r}\right)$, als auch für die links davon $\left(A_{2 l}\right)$. Bei $C_{2}$ handelt es sich nicht um die im Brunnen gemessene Konzentration $C_{w}\left(t_{2}\right)\left(C_{n} \neq C_{w}\left(t_{n}\right)\right.$ für $\left.t_{n}>t_{1}\right)$, da $C_{w}\left(t_{2}\right)$ eine Mischkonzentration aus $C_{1}$ und $C_{2}$ ist. Diese Prozedur wird für jede weitere Isochrone sequenziell wiederholt, wobei jeweils die Konzentrationen der vorangegangenen Isochronen berücksichtigt werden. Details sind z. B. in Bayer-Raich et al. (2003) und Bayer-Raich (2004) enthalten, während eine ausführlichere Beschreibung der IPVs bei Ptak et al. (2000) zu finden ist.

\section{Konditionierung}

Die Inversion der Konzentrationsganglinien ist in der Regel nicht eindeutig, da es verschiedene Möglichkeiten der Schadstoffverteilung im Grundwasserleiter gibt, welche die gemessene Konzentrationsganglinie darstellen könnte (z. B. Jarsjö et al. 2005). Der Inversionsalgorithmus erfüllt dabei die Massenerhaltungsgleichung (Gl. 2), kann aber nicht genau definieren wie die Masse im Grundwasserleiter entlang der Kontrollebene verteilt ist. Um die verschiedenen Möglichkeiten zunächst einzugrenzen, kann man sich folgende Szenarien vorstellen: die im Brunnen gemessene Konzentration, z.B. $C_{w}\left(t_{2}\right)$, ergibt für $A_{2 r}$ und $A_{2 l}$ erstens entweder eine Konzentration von 0 bzw. $2 C_{2}$, zweitens von jeweils $C_{2}$ (Abb. 2) oder drittens von $2 C_{2}$ bzw. 0. Diese drei Varianten der Schadstoffverteilung in der Fahne sind in CSTREAM implementiert und werden dort als (in Richtung Unterstrom betrachtet) linksseitige, symmetrische (Abb. 2) und rechtsseitige Lösung bezeichnet.

Diese Nichteindeutigkeit wirft die Frage nach der tatsächlichen Fahnenposition auf. Eine Konditionierung der CSTREAM-Inversionslösung mittels Punktkonzentrationsmessungen kann helfen, diese näher einzugrenzen. Nach einer Erweiterung bietet CSTREAM die Möglichkeit, sogenannte „Constant Concentration Zones“ (CCZ) zu definieren. Diese „Constant Concentration Zones“ haben jeweils den Umfang von mindestens einer Stromröhre (Abb. 2) im natürlichen Grundwasserströmungsfeld und sind für Bereiche im Einzugsgebiet eines IPV repräsentativ, in denen z.B. mittels Direct-Push-Verfahren oder in Messstellen
Punktkonzentrationswerte für die untersuchten Schadstoffe bestimmt worden sind. Durch Überlagern einer georeferenzierten Karte des Untersuchungsgebietes (inklusive DirectPush-Messstellen) mit den georeferenzierten Stromröhren aus CSTREAM wird bestimmt, welche Stromröhre welche Punktkonzentrationsmessung repräsentiert.

Diese werden als CCZ räumlich definiert und CSTREAM die CCZ-Position und die gemessenen Punktkonzentrationswerte vorgegeben. Bei der konditionierten Auswertung berücksichtigt das erweiterte CSTREAM die CCZ mit konstanter Konzentration, d.h. mit der gemessenen Punktkonzentration, und gleicht nur die Konzentration die der CCZ gegenüberliegenden Stromröhre aus, wobei die Massenerhaltung entsprechend Gleichung 2 eingehalten wird. Bei allen anderen Stromröhren wird der symmetrische Fall (s. Abb. 2) angenommen.

\section{Durchführung}

Auf dem Gelände des Testfeldes Süd befinden sich 4 IPVKontrollebenen (Abb. 3), die alle etwa im rechten Winkel zur vorherrschenden Grundwasserfließrichtung positioniert sind und einen Abstand von $140 \mathrm{~m}$ (CP1), $240 \mathrm{~m}$ (CP2-Ersatz), $330 \mathrm{~m}$ (CP2-3) und $450 \mathrm{~m}$ (CP3 neu) zur vermuteten Schadstoffquelle haben. Nach IPVs in den Jahren 2003 (CP2-Ersatz) und 2004 (CP3), wurden im Jahr 2006 acht neue IPVs an den Brunnen der CP2-3 (B97-B102), sowie an B86 und B96 innerhalb eines Zeitraums von 42 Tagen durchgeführt. Langjährige Untersuchungen auf dem Gelände führen zu der Annahme, dass es sich um eine stationäre Fahne handelt, wodurch der zeitliche Abstand zwischen den einzelnen IPVs eine geringe Bedeutung hat. Um gegenseitige Beeinflussung zu vermeiden, wurden nur maximal 2 Brunnen gleichzeitig bepumpt. Des Weiteren wurde eine möglichst lange Wiederanstiegsphase gewählt (3 Tage), um eine Wiederherstellung des natürlichen Strömungsfeldes zu ermöglichen, was durch fortlaufende Wasserstandsmessungen kontrolliert wurde.

Die Proben wurden auf HET, PAK, BTEX und Ionen untersucht. Der Temperaturverlauf, die elektrische Leitfähigkeit, der pH-Wert, das Redoxpotenzial und die Konzentration an gelöstem Sauerstoff wurden mithilfe einer Durchflusszelle im Förderstrom der IPV-Brunnen bestimmt. Für die BTEX-Bestimmung wurden $100 \mathrm{ml}$-Braunglasflaschen mit Wasser vollständig befüllt, luftdicht verschlossen (Teflondichtung) und bis zur Messung, wie alle anderen Wasserproben, bei $4{ }^{\circ} \mathrm{C}$ gelagert. Die BTEX-Analyse der Proben erfolgte innerhalb von 24 Stunden an einem GC-MS-System (HP 6890; MSD 5972 A von HP) das mit einem Purge\&TrapSystem (Tekmar ALS 2016, Tekmar 3000) gekoppelt ist. Für die PAK-Bestimmung, wurden Wasserproben mit Lösungsmittel extrahiert und die Extrakte mit GC-MS (HP 5890; MSD 5972 von HP) analysiert. Die zur Anreicherung von HET aus Wasser eingesetzten SPE-Kartuschen wurden 
Abb. 3 Testfeld Süd mit Modellbereich und Randbedingungen sowie Position der Kontrollebenen und Brunnen.

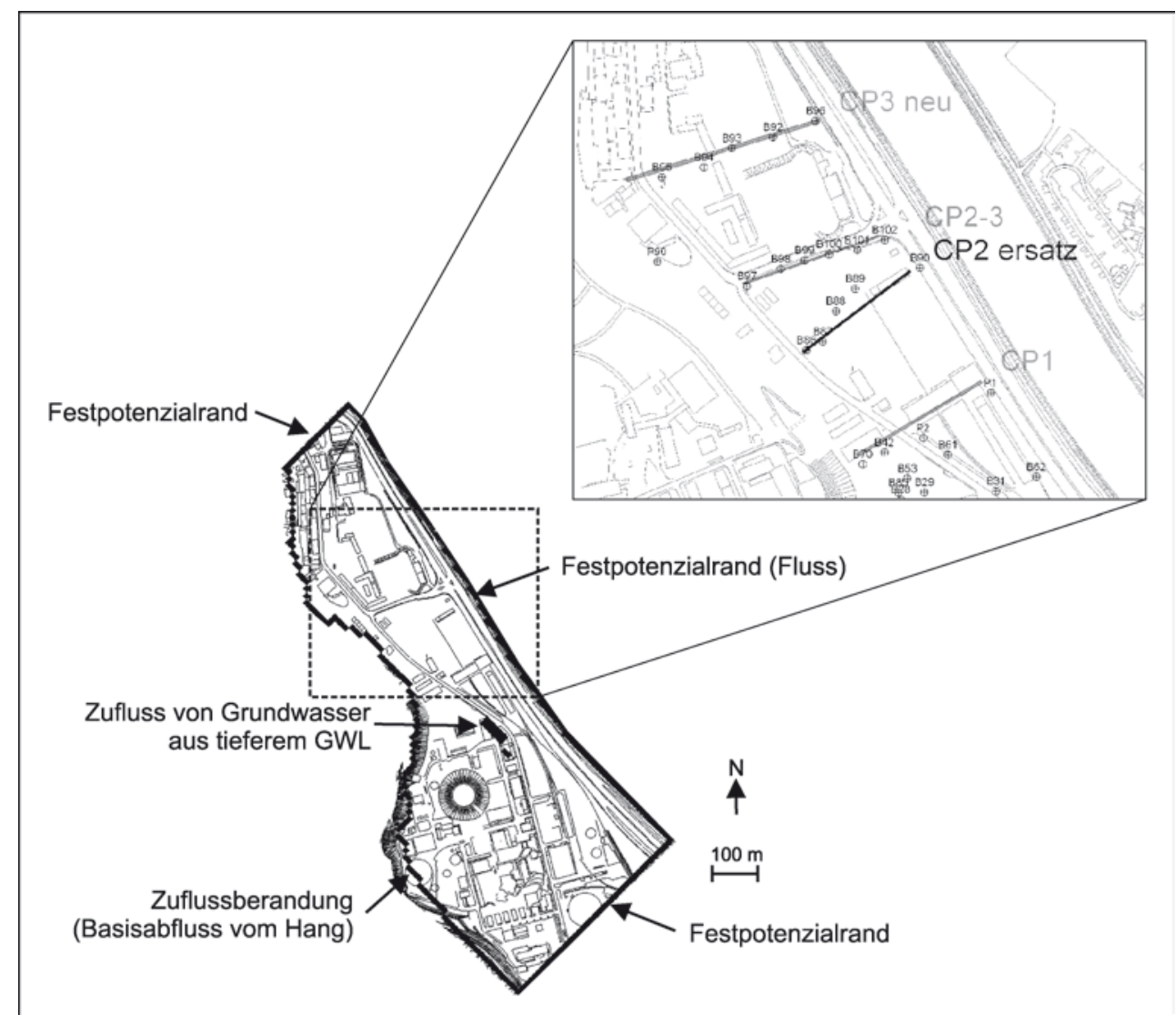

mit Lösungsmittel eluiert, und die eingeengten Eluate mit GC-MS (HP 5890; MSD 5972 von HP) analysiert. Anionen und Kationen der Proben wurden mit einem Ionenchromatograph (DX-120 von Dionex) bzw. Atomabsorptions-Spektrometer (Perkin Elmer 1100) bestimmt.

Des Weiteren ist zu beachten, dass nur im Fall eines Retardationsfaktors $R=1$ die Erfassungsbereiche im Hinblick auf das Grundwasser und die Schadstoffe identisch sind. Im Falle von $R>1$ ist der Erfassungsbereich für den Schadstoff kleiner als für das Grundwasser. Im Zuge des Projektes wurden mithilfe von Säulenversuchen, welche Material ähnlich dem des Testfeld Süd enthielten, Retardationsfaktoren für die Schadstoffe Acenaphthen, 2-Methylbenzofuran und 2,3-Dimethylbenzofuran bestimmt. Die Probennahmezeiten der Konzentrationsganglinien wurden deshalb entsprechend der Vorgehensweise in Bockelmann et al. (2001) mit den folgenden Retardationsfaktoren angepasst: $R_{A c e}=2,8$, $R_{M B F}=1,2$ und $R_{D M B F}=1,96$.

Zur Konditionierung der numerischen Inversionslösung in CSTREAM wurden 13 Direct-Push-Messstellen im Abstand von 3 bis 11 Meter zwischen den Brunnen B97 und B99 errichtet (Abb. 4). Eine solche Punktmessung der Schadstoffkonzentration an einer Direct-Push-Messstelle repräsentiert im Schnitt eine Stromröhre mit einer Breite von $2 \mathrm{~m}$. Die Grundwasserproben aus diesen Messstellen wurden auf die- selben Schadstoffe wie bei den Immissionspumpversuchen untersucht, um so Punktkonzentrationswerte zu erhalten.

Bisherige Modellierung von Strömung und Transport

Im Zuge der bisherigen Untersuchungen im Testfeld Süd wurden mehrere, teilweise aufeinander aufbauende hydraulische Modelle vom zentralen und südlichen Teil entwickelt (Bockelmann et al. 2001, Bockelmann et al. 2003, Mak et al. 2006). Des Weiteren wurde ein konservatives Transportmodell in Verbindung mit der Analyse der Kohlenstoffisotopenverhältnisse von Benzol, Toluol und O-Xylol zur Bestimmung des In-Situ-Abbaus entwickelt (Mak et al. 2006).

Das in dieser Studie zur Inversion der IPV-Konzentrationsganglinien mittels CSTREAM verwendete numerische Standortmodell basiert auf den bereits erwähnten Modellen, insbesondere auf dem von Mak et al. (2006), und wurde in Richtung Norden erweitert, um die Schadstofffahne und alle Kontrollebenen abzudecken (Abb. 3). Es handelt sich um ein mit MODFLOW (Harbaugh \& McDonald 1996) und MODPATH (Pollock 1994) erstelltes zweidimensionales Modell. Ein 2D-Ansatz wurde gewählt, da der Grundwasserleiter geringmächtig und die Brunnen voll verfiltert sind. Dieses 2DModell besteht aus einer gespannten/ungespannten Schicht mit 190 Zeilen und 216 Spalten mit einer Zellgröße von $5 \mathrm{~m}$ 


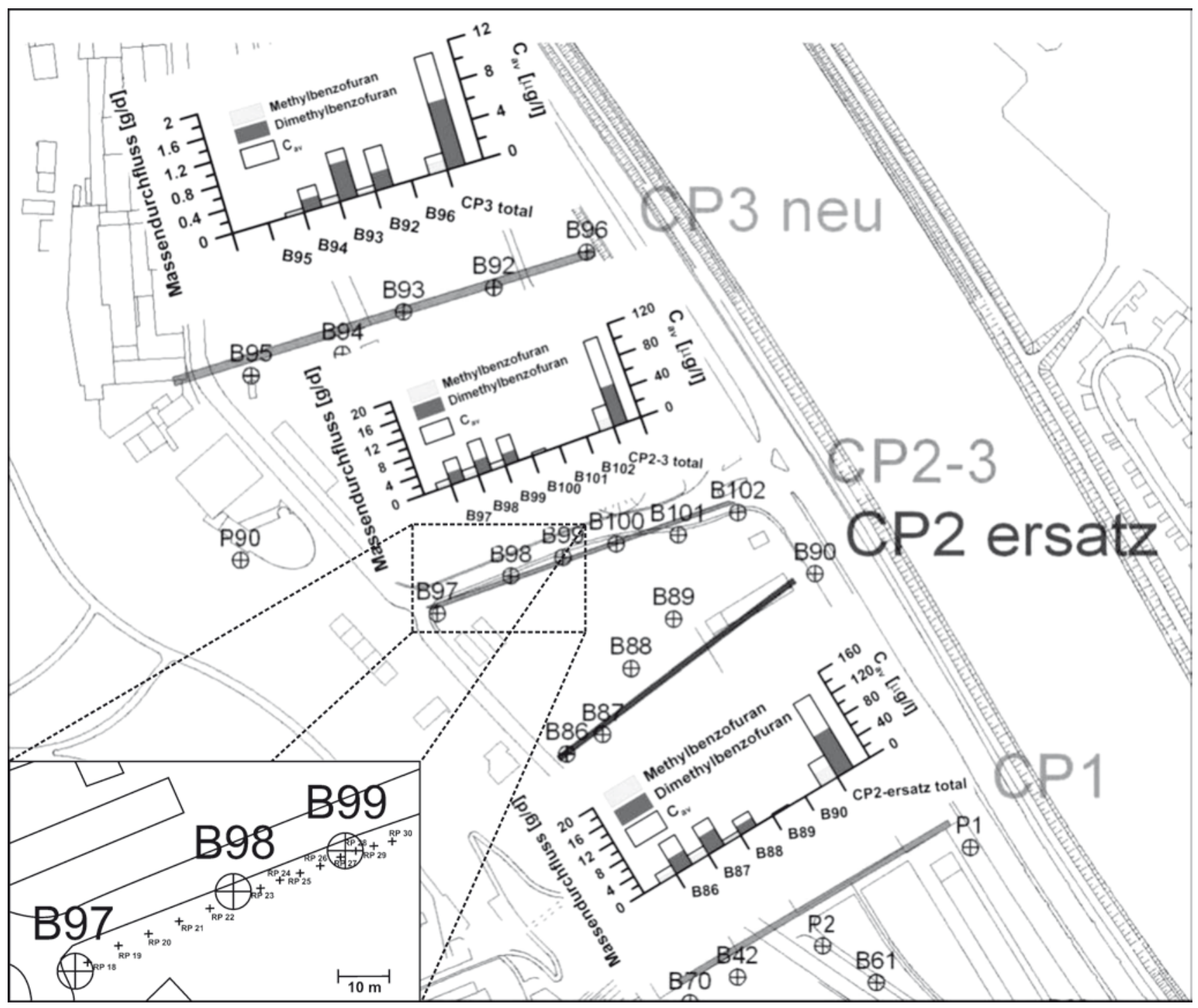

Abb. 4 Schadstoffmassenfrachten und mittlere Konzentration $\left(\mathrm{C}_{\mathrm{AV}}\right)$ an den 3 Kontrollebenen (IPV-spezifische Auftragung und Summe der Frachten, jeweils rechts aufgetragen) sowie Position der Direct-Push-Messstellen zur Konditionierung der numerischen Inversionslösung mittels Punktkonzentrationswerten (Vergrößerung).

mal $5 \mathrm{~m}$ mit lokalen Verfeinerungen (bis maximal $1 \mathrm{~m}$ mal $1 \mathrm{~m}$ an den Brunnen). Die verwendeten Randbedingungen sind in Abbildung 3 dargestellt. Potenzialmessungen im Feld (im Rahmen von Stichtagsmessungen) ergaben nur geringe Grundwasserstands- und Fließrichtungsänderungen, was sich auf die versiegelte Oberfläche im Untersuchungsgebiet und den regulierenden Einfluss des gestauten Vorfluters zurückführen lässt. Dies führt zu der Annahme, dass es sich um ein stationäres System handelt. Der Randzufluss im zentralen und südlichen Teil wurde von Mak et al. (2006) übernommen.

\section{Ergebnisse}

Tabelle 1 stellt die ermittelten Schadstofffrachten an den drei untersuchten Kontrollebenen für die im Testfeld Süd dominierenden Schadstoffkomponenten dar, die mithilfe der Software CSTREAM und dem numerischen Standortmodell für das Gelände berechnet wurden.

Die Gesamtschadstofffracht an den einzelnen Kontrollebenen nimmt von Süden nach Norden, d.h. mit größerer Entfernung zum vermuteten Schadensherd, ab. Dabei ist die Abnahme von CP2-Ersatz zu CP2-3 (Abschnitt 1) deutlich geringer als von CP2-3 zu CP3 neu (Abschnitt 2). Dies wird insbesondere bei den Methylbenzofuranen (Abnahme: 0,64 $\mathrm{g} \mathrm{d}^{-1}$ für Abschnitt 1 und 2,01 $\mathrm{g} \mathrm{d}^{-1}$ für Abschnitt 2) und Dimethylbenzofuranen (Abnahme: $1,11 \mathrm{~g} \mathrm{~d}^{-1}$ bzw. 7,16 $\mathrm{g} \mathrm{d}^{-1}$ ) deutlich. Acenaphthen zeigt einen kleineren Unterschied (Abnahme: 4,9 $\mathrm{g} \mathrm{d}^{-1}$ bzw. 10,73 $\mathrm{g} \mathrm{d}^{-1}$ ). Es wird weiterhin deutlich, dass wie schon bei Vorarbeiten zum laufenden Projekt festgestellt wurde, die HETs im Abschnitt 1 weniger 
Tab. 1 Schadstofffracht an CP2-Ersatz, CP2-3 und CP3 neu; n. n. = nicht nachweisbar.

\begin{tabular}{|c|c|c|c|c|c|c|c|}
\hline CP2-Ersatz [g d $\left.{ }^{-1}\right]$ & B86 & B87 & B88 & B89 & B90 & Total & \\
\hline Acenaphthen & 8,1 & 5,92 & 1,61 & 0,000539 & 0,000813 & 15,63 & \\
\hline Methylbenzofurane & 1,01 & 1,24 & 0,563 & 0,0113 & n. n. & 2,82 & \\
\hline Dimethylbenzofurane & 3,41 & 3,98 & 1,95 & 0,0403 & n. n. & 9,38 & \\
\hline CP2-3 [g d $\left.{ }^{-1}\right]$ & B97 & B98 & B99 & B100 & B101 & B102 & Total \\
\hline Acenaphthen & 3,18 & 5,29 & 2,23 & 0,0196 & 0,000822 & 0,00631 & 10,73 \\
\hline Methylbenzofurane & 0,706 & 0,86 & 0,565 & 0,0454 & n. n. & n. n. & 2,18 \\
\hline Dimethylbenzofurane & 2,54 & 3,04 & 2,45 & 0,236 & n. n. & n. n. & 8,27 \\
\hline CP3 neu $\left[\mathrm{g} \mathrm{d}^{-1}\right]$ & B95 & B94 & B93 & B92 & B96 & Total & \\
\hline Acenaphthen & n. $n$. & n. $n$. & 0,00237 & 0,000779 & 0,000408 & 0,0036 & \\
\hline Methylbenzofurane & n. n. & 0,0385 & 0,0887 & 0,0417 & n. n. & 0,17 & \\
\hline Dimethylbenzofurane & n. n. & 0,203 & 0,618 & 0,292 & n. n. & 1,11 & \\
\hline
\end{tabular}

stark abnehmen als die PAKs, also eine wie hier geplante und eingesetzte Sanierungsmaßnahme insbesondere für die HETs nötig ist.

Entlang der einzelnen Kontrollebenen lässt sich ebenfalls ein deutlicher Trend ausmachen, wobei sich der Hauptstrom der Schadstoffe auf ein bis drei Brunnen konzentriert. An CP2-Ersatz handelt es sich dabei um die Messstellen B86 bis B89 mit der Hauptfracht an B86. Die zweite Kontrollebene CP2-3 zeigt die höchste Schadstofffracht an den Messstellen B97 bis B99 mit dem Maximum an B98. Die dritte Kontrollebene (CP3 neu) ist durch sehr geringe Konzentrationen, und damit Frachten, aller Schadstoffe gekennzeichnet, was eine eindeutige Bestimmung von Trends schwierig macht. Bereits die IPVs im Jahr 2003 (CP2-Ersatz) lokalisierten die Hauptfracht an der Messstelle B86, woraus sich schlussfolgern lässt, dass sich das Zentrum der Fahne bei B86 befindet und sich Richtung Norden bis B98 fortsetzt. Die an B86 und B98 angrenzenden Messstellen (B87 und B88 bzw. B97 und B99) deuten auf eine vom Zentrum der Fahne Richtung Berghang bzw. Fluss hin abnehmenden Schadstoffmassenfluss und die Ausbildung einer einzelnen zusammenhängenden Fahne von Acenaphthen, Methylbenzofuranen und Dimethylbenzofuranen.

Die Auswertung der IPVs hat gezeigt, dass entlang einer Kontrollebene die höchste (für einen einzelnen IPV repräsentative) mittlere Konzentration $C_{A V}$ (definiert als Schadstoffmassenfracht bezogen auf den Grundwasserabfluss) nicht notwendigerweise mit der größten absoluten Schadstoffmassenfracht zusammenfallen muss (Abb. 4, insbesondere B86 und B87 an CP2-Ersatz). Das lässt sich durch den Einfluss der räumlich variablen Grundwasserströmung im Grundwasserleiter, hervorgerufen durch die heterogene Verteilung der hydraulischen Durchlässigkeit, erklären.

Die entlang der einzelnen Stromröhren mit CSTREAM berechneten Konzentrationen für die Messstellen B97, B98 und B99 sind exemplarisch in Abbildung 5a, c und e dargestellt. Hierbei wurde zunächst die symmetrische Inversionslösung (symmetrisch um jede der drei Messstellen) gewählt, wobei sich die jeweils außen liegenden Stromröhren nicht mit denen der angrenzenden Messstellen überschneiden. Die durch die unkonditionierte Inversionslösung bewirkte Symmetrie ist deutlich erkennbar, wobei in diesem Fall, bedingt durch die Auswahl der Brunnen, bereits die unkonditionierte Lösung deutlich das postulierte Fahnenzentrum an B98 hervorhebt, wenn man die Inversionsergebnisse an B97, B98 und B99 vergleichend betrachtet.

Die Resultate der Konditionierung der numerischen Inversionslösung mittels Punktkonzentrationsmesswerten aus den Direct-Push-Messstellen sind in Abbildung 5b, $\mathrm{d}$ und $\mathrm{f}$ dargestellt. Insbesondere kann die Konditionierung hier helfen, die Fahnenränder genauer einzugrenzen, da die Konzentrationen aus der unkonditionierten symmetrischen Lösung hierbei teilweise zum Rand hin sogar ansteigen (vgl. Abb. 5a). In diesem Fall zeigt die konditionierte Lösung (Abb. 5b), dass der östliche Fahnenrand bei B99 besser eingegrenzt werden konnte als der westliche Rand bei B97, da sich dort weniger Direct-Push-Messstellen befinden als bei B99. Das führt dazu, dass westlich von Messstelle B97 und östlich von Messstelle B99 bzw. RP30 die Konzentrationen nach der Konditionierung entweder die Werte der symmetrischen Lösung beibehalten oder durch den beschriebenen Massenerhalt ansteigen bzw. sinken. Ein ähnlicher Effekt lässt sich auch bei den Methylbenzofuranen und Dimethylbenzofuranen (Abb. 5d, f) beobachten.

Die in der Nähe des postulierten Fahnenzentrums in den Direct-Push-Messstellen gemessenen Konzentrationen liegen bei allen drei Schadstoffen, bis auf jeweils eine Ausnahme (RP22 bzw. RP23), auf deutlich höherem Niveau als in den Randbereichen. Damit wird die Annahme eines Fahnenzentrums um B98 bestätigt. 
Abb. 5

Resultate der symmetrischen (a, c und e) und konditionierten $(b$, d und f) IPVAuswertung: Konzentrationsprofile an der Kontrollebene CP2-3; Abstände relativ zum IPV-Brunnen B98 sind in a, $c$ und e dargestellt.

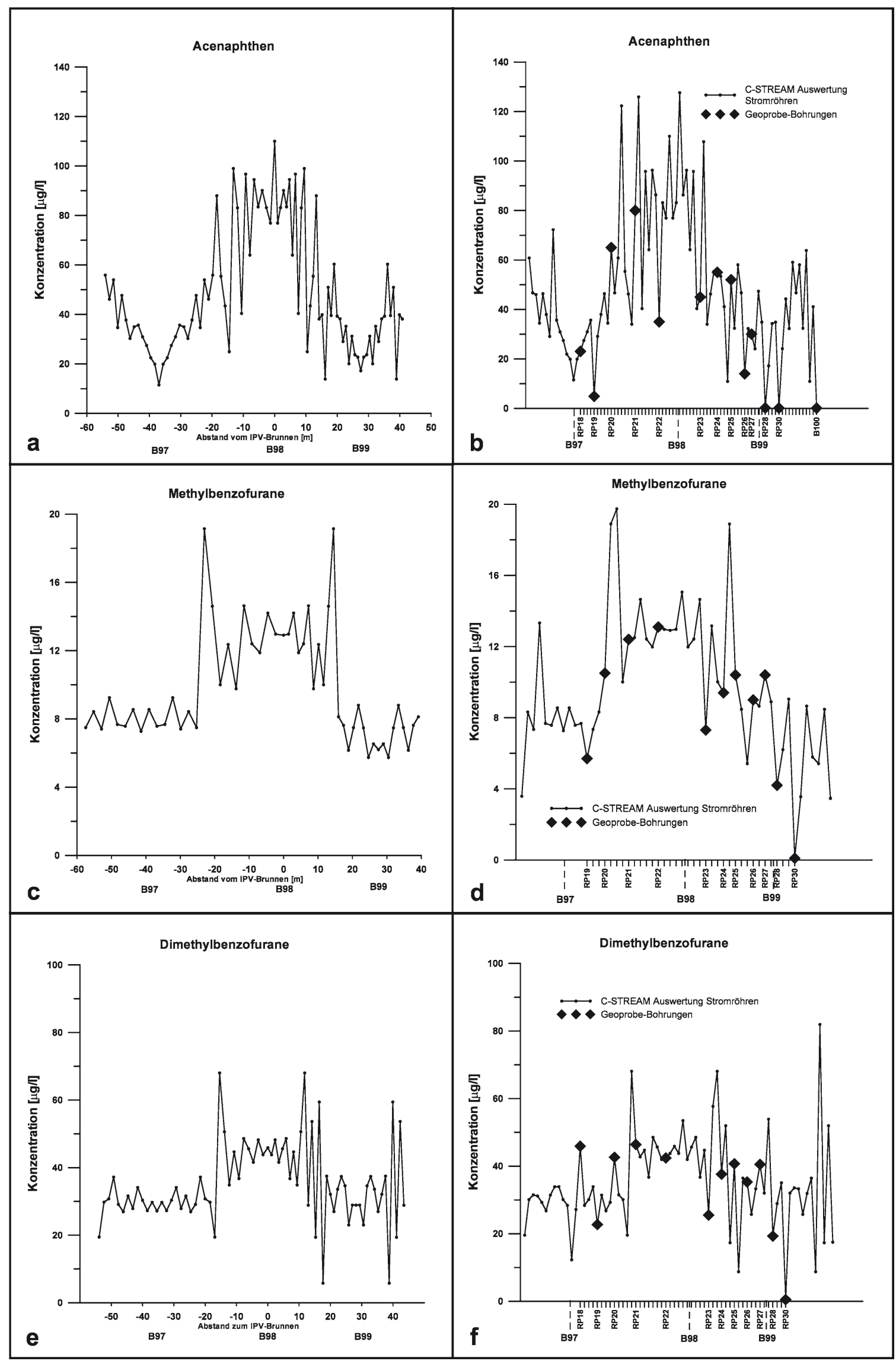




\section{Schlussfolgerungen und Ausblick}

Immissionspumpversuche leisten, wie schon in vorangegangenen Studien deutlich gemacht wurde (Bockelmann et al. 2001, Jarsjö et al. 2005, Ptak et al. 2004), einen wertvollen Beitrag zur sicheren Berechnung von Schadstofffrachten und Konzentrationsverteilungen an Kontrollebenen. Angewendet an mehreren, in Abstromrichtung aufeinanderfolgend angeordneten Kontrollebenen erlaubt die integrale Natur der Methode in der Regel eine deutlich genauere und eindeutigere Bestimmung von Natural-Attenuation-Raten im Vergleich zu Ansätzen, die auf Interpolation von Punktkonzentrationsmessungen basieren. Die integrale Methode leistet damit einen wichtigen Beitrag zum Nachweis und der Implementierung von Natural Attenuation an einem Standort. Hierbei hilft die Anwendung eines numerischen Strömungsund Transportmodells, durch das es möglich wird, auch hydraulisch heterogene Standortbedingungen zu simulieren. Bislang konnte allerdings nur die ungefähre Position der Schadstofffahne entlang von Kontrollebenen bestimmt werden. Die hier beschriebene Konditionierung der numerischen IPV-Inversionslösung mit Punktkonzentrationsmesswerten, z.B. aus Direct-Push-Messstellen, erlaubt es, diese Position näher einzugrenzen und die Nichteindeutigkeit bzw. Unsicherheit bei der inversen Bestimmung von Schadstofffrachten und der räumlichen Konzentrationsverteilung entlang von Kontrollebenen signifikant zu reduzieren. Ebenso wird die Aussagesicherheit von Backtracking-Verfahren zur Eingrenzung von Schadensherden (Jarsjö et al. 2005) signifikant verbessert. Diese Erhöhung der Erkundungsgenauigkeit ist insbesondere bei der Planung und Durchführung von Sanierungsmaßnahmen hilfreich.

Die Konditionierung macht für das Testfeld Süd deutlich, dass mehr Informationen aus den Randbereichen des Untersuchungsgebiets nötig sind, um insbesondere westlich von B97 genauere Aussagen treffen zu können.

Die Ergebnisse der IPV-Auswertung sollen des Weiteren bei der reaktiven Transportmodellierung im Testfeld Süd eingesetzt werden, um die Schadstoffverteilung zur Dimensionierung einer innovativen Sanierungsmaßnahme (Enhanced Natural Attenuation unter $\mathrm{H}_{2} \mathrm{O}_{2}$-Einsatz) als Randbedingungen zu nutzen. Zusätzlich sollen die Ergebnisse der konditionierten IPV-Auswertung einen Beitrag zum Eingrenzen der Position des Schadensherdes mittels Backtracking leisten.

Danksagung Das diesem Bericht zugrundeliegende Vorhaben wurde mit Mitteln des Bundesministeriums für Bildung und Forschung (BMBF) unter dem Förderkennzeichen 02WN0361 im Rahmen des Projektverbundes KORA gefördert. Die Autoren bedanken sich für die Finanzierung durch das BMBF sowie für die freundliche Unterstützung durch das UFZ Leipzig-Halle GmbH (Martí Bayer-Raich), durch die Firma Büro Holder und Herrn Matthias Piepenbrink für seine Mitarbeit in diesem Projekt.
OpenChoice Dieser Artikel wird zu den Bedingungen der „Creative Commons Attribution Noncommercial License“ zur Verfügung gestellt. Damit ist eine nichtkommerzielle Nutzung, Verbreitung und Vervielfältigung erlaubt, sofern die Autoren des Artikels und die genaue Quelle angegeben sind.

\section{Literatur}

Bauer, S., Bayer-Raich, M., Holder, T., Kolesar, C., Müller, D., Ptak, T.: Quantification of groundwater contamination in an urban area using integral pumping tests.- J. Contam. Hydrol. 75, 183-213 (2004)

Bayer-Raich, M.: Integral pumping tests for the characterization of groundwater contamination. PhD Thesis, Center for Applied Geoscience, Universität Tübingen.- 112 S.; Tübingen (2004)

Bayer-Raich, M., Jarsjö, J., Holder, T., Ptak, T.: Numerical estimations of contaminant mass flow rate based on concentration measurements in pumping wells. Calibration and Reliability in Groundwater Modelling: A Few Steps Closer to Reality (Proceedings of ModelCARE'2002, Prague, Czech Republic, June 2002).- IAHS Publ. No. 277, 10-16 (2003)

Bayer-Raich, M., Jarsjö, J., Liedl, R., Ptak, T., Teutsch, G.: Average contaminant concentration and mass flow in aquifers from time dependent pumping well data: analytical framework.- Water Resour. Res. 40, W08303, doi:10.1029/2004/WR003095 (2004)

Bayer-Raich, M., Jarsjö, J., Liedl, R., Ptak, T., Teutsch, G.: Integral pumping test analyses of linearly sorbed groundwater contaminants using multible wells: Inferring mass flows and Natural Attenuation rates.- Water Resour. Res. 42, W08411, doi:10.1029/2005WR004244 (2006)

Bockelmann, A., Ptak, T., Teutsch, G.: An analytical quantification of mass fluxes and natural attenuation rate constants at a former gasworks site.- J. Contam. Hydrol. 53(3-4), 429-453 (2001)

Bockelmann, A., Zamfirescu, D., Ptak, T., Grathwohl, P., Teutsch, G.: Quantification of mass fluxes and natural attenuation rates at an industrial site with a limited monitoring network: a case study.J. Contam. Hydrol. 60, 97-121 (2003)

Bösel, D., Herfort, M., Ptak, T., Teutsch, G.: Design, performance, evaluation and modelling of a natural gradient multitracer transport experiment in a contaminated heterogeneous porous aquifer.- In: Dassargues, A. (Editor), Tracers and Modelling in Hydrogeology.- IAHS Publication, Liege 262, 45-51 (2000)

Borden, R.C., Daniel, R.A., Lebrun IV, L.E., Davis, C.W.: Intrinsic biodegradation of MTBE and BTEX in a gasoline-contaminated aquifer.- Water Resour. Res. 33(5), 1105-1115 (1997)

Chiang, C.Y., Salanitro, J.P., Chai, E.Y., Colthart, J.D., Klein, C.L.: Aerobic biodegradation of benzene, toluene, and xylene in a sandy aquifer - Data analysis and computer modeling.- Ground Water 27(6), 823-834 (1989)

Gieg, L.M., Kolhatkar, R.V., McInerney, M.J., Tanner, R.S., Harris Jr, S.H., Sublette, K.L., Suflita, J.M.: Intrinsic Bioremediation of Petroleum Hydrocarbons in a Gas Condensate-Contaminated Aquifer.- Environ. Sci. Technol. 33, 2550-2560 (1999)

Harbaugh, A.W., McDonald, M.G.: User's documentation for MODFLOW-96, an update to the U.S. Geological Survey modular finite-difference ground-water flow model.- U.S. Geological Survey Open-File Report 96-485 (1996)

Herfort, M., Ptak, T., Hümmer, O., Teutsch, G., Dahmke A.: Testfeld Süd: Einrichtung der Testfeldinfrastruktur und Erkundung hydraulisch-hydrogeochemischer Parameter des Grundwasserleiters.- Grundwasser 3(4), 159-166 (1998)

Herfort M.: Reactive transport of organic compounds within a heterogeneous porous aquifer. $\mathrm{PhD}$ Thesis, Universität Tübingen.59 S.; Tübingen (2000)

Hunkeler, D., Aravena, R., Butler, B.J.: Monitoring microbial dechlorination of tetrachloroethene (PCE) in groundwater using com- 
pound-specific stable carbon isotope ratios: microcosm and field studies.- Environ. Sci. Technol. 33(16) 2733-2738 (1999)

Jarsjö, J., Bayer-Raich, M., Ptak, T.: Monitoring groundwater contamination and delineating source zones at industrial sites: Uncertainty analyses using integral pumping tests.- J. Contam. Hydrol. 79, 107-134 (2005)

King, M.W.G., Barker J.F., Devlin, J.T., Butler, B.J.: Migration and natural fate of a coal tar creosote plume: 2 . Mass balance and biodegradation indicators.- J. Contam. Hydrol. 39, 281-307 (1999)

Mak, K.S., Griebler, C., Meckenstock, R.U., Liedl, R., Peter, A.: Combined application of conservative transport modelling and compound-specific carbon isotope analyses to assess in situ attenuation of benzene, toluene, and o-xylene.- J. Contam. Hydrol. 88, 306-320 (2006)

Marotz, G.: Technische Grundlagen einer Wasserspeicherung im natürlichen Untergrund.- 228 S.; Verl. Wasser u. Boden, Hamburg (1968)

Nyer, E.K., Duffin, M.E.: The state of the art of bioremediation.Ground Water Monit. R. 17(2), 64-69 (1997)

Pollock, D.: User's guide for MODPATH/MODPATH-PLOT, Version 3: A particle tracking post-processing package for MODFLOW, the U.S. Geological Survey finite-difference ground-water flow model.- U. S. Geological Survey (1994)

Ptak T., Schwarz, R., Holder, T., Teutsch, G.: Ein neues integrales Verfahren zur Quantifizierung der Grundwasserimmission: II. Numerische Lösung und Anwendung in Eppelheim.- Grundwasser 5(4), 176-183 (2000)
Ptak, T., Bayer-Raich, M., Bauer, S.: Tiefenorientierte integrale Erkundung der Schadstoffbelastung in großräumig kontaminierten Aquiferen.- Grundwasser 9(4), 235-247 (2004)

Rügner, H., Holder, T., Maier, U., Bayer-Raich, M., Grathwohl, P., Teutsch, G.: Natural Attenuation- Untersuchungen ,ehemalige Abfalldeponie Osterhofen.- Grundwasser 9(2), 98-108 (2004)

Schwarz, R.: Grundwasser-Gefährdungsabschätzung durch Emissions- und Immissionsmessungen an Deponien und Altlasten. $\mathrm{PhD}$ thesis, Center of Applied Geosciences, Universität Tübingen.125 S.; Tübingen (2002)

Teutsch, G., Ptak, T., Schwarz, R., Holder, T.: Ein neues integrales Verfahren zur Quantifizierung der Grundwasserimmission: I. Theoretische Grundlagen.- Grundwasser 5(4), 170-175 (2000)

Therrin, J., Davis, G.B., Barber, C.: A ground-water tracer test with deuterated compounds for monitoring in situ biodegradation and retardation of aromatic hydrocarbons.- Ground Water 33(3), 469-475 (1995)

Wiedemeier, T.H., Swanson, M.A., Wilson, J.T., Kampbell, D.H., Miller, R.N., Hansen, J.E.: Approximation of biodegradation rate constants for monoaromatic hydrocarbons (BTEX) in groundwater.- Ground Water Monit. R. 16(3), 186-194 (1996)

Zamfirescu, D.: Release and fate of specific organic contaminants at a former gasworks site. PhD Thesis, Universität Tübingen.- 96 S.: Tübingen. (2000)

Zamfirescu, D., Grathwohl, P.: Occurrence and attenuation of specific organic compounds in the groundwater plume at a former gasworks site.- J. Contam. Hydrol. 53(3-4), 407-427 (2001) 